\title{
Acoustic Leading-Edge Receptivity for \\ Supersonic/Hypersonic Flows over a Blunt Wedge
}

\author{
Adriano Cerminara*, Neil D. Sandham ${ }^{\dagger}$ \\ Aerodynamics and Flight Mechanics Research Group \\ University of Southampton, Southampton, Hampshire, SO17 1BJ, United Kingdom
}

Leading-edge receptivity to fast and slow acoustic waves of boundary layers on a cylinderwedge geometry is investigated for a set of six different cases with Mach number ranging from 3.0 to 7.3, through direct numerical simulations of the Navier-Stokes equations. The structure of the disturbance field transmitted downstream of the shock by the imposed freestream waves is analyzed, as well as the characteristics of the wall response and its sensitivity to the angle of attack and the freestream-wave inclination angle. The results show that different post-shock wave structures are formed for fast and slow acoustic waves, consisting of high-amplitude dragged and reflected waves for the fast-wave case, and of low-amplitude convected waves for the slow-wave case. A good agreement is found with linear interaction theory. The wall response along the wall for fast waves shows a strong resonant amplification of mode $F$ in the nose region, and a modulated long-wavelength behavior further downstream. In contrast, the response to slow waves shows an initial decay in the leading-edge region, and an overall lower amplitude. The simulation results enable freestream disturbances, which are difficult to measure directly in experiments, to be related to wall pressure fluctuations.

* Post Graduate Research Student, Student Member AIAA

${ }^{\dagger}$ Professor of Aerospace Engineering 


\section{Nomenclature}

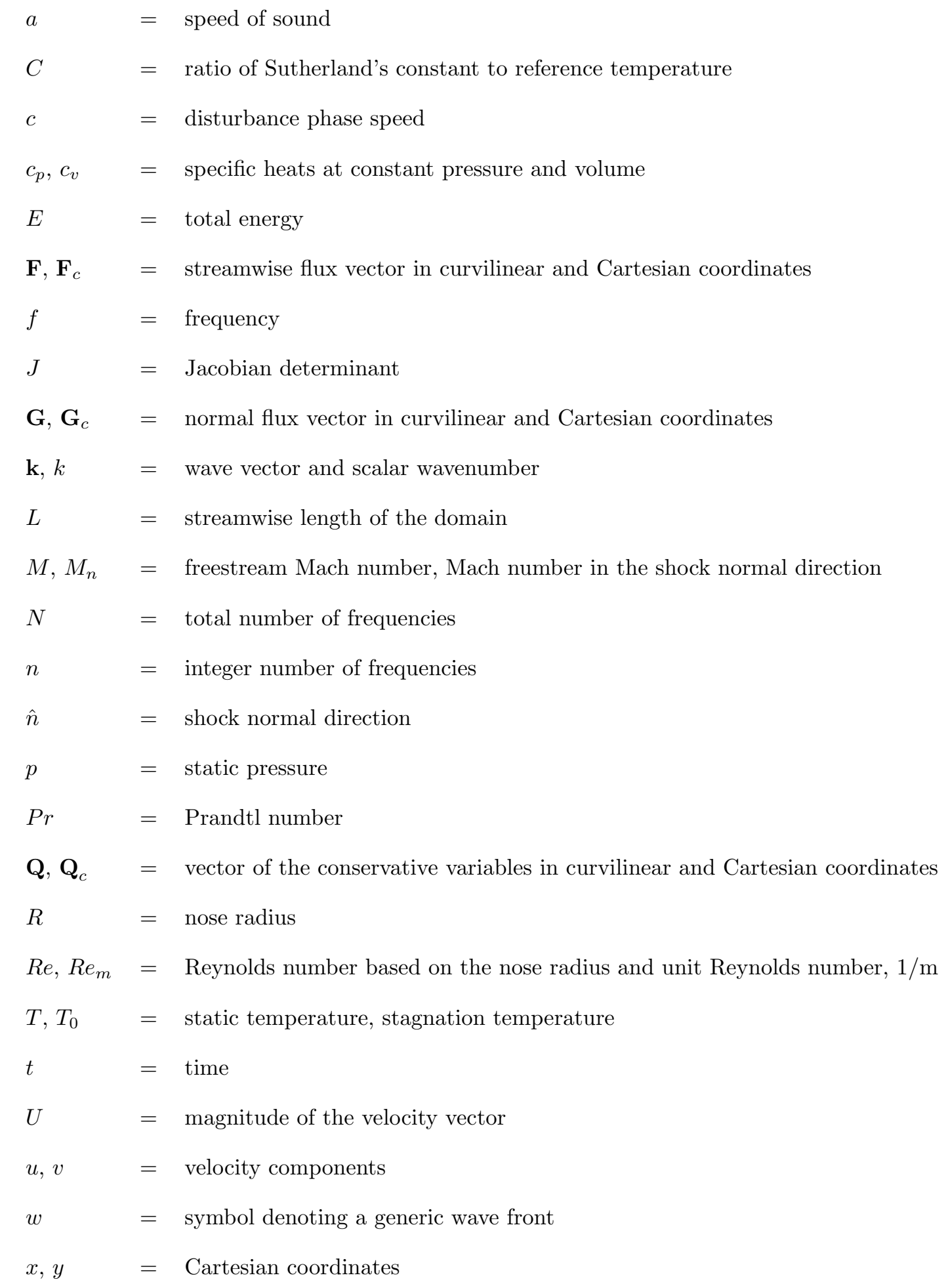




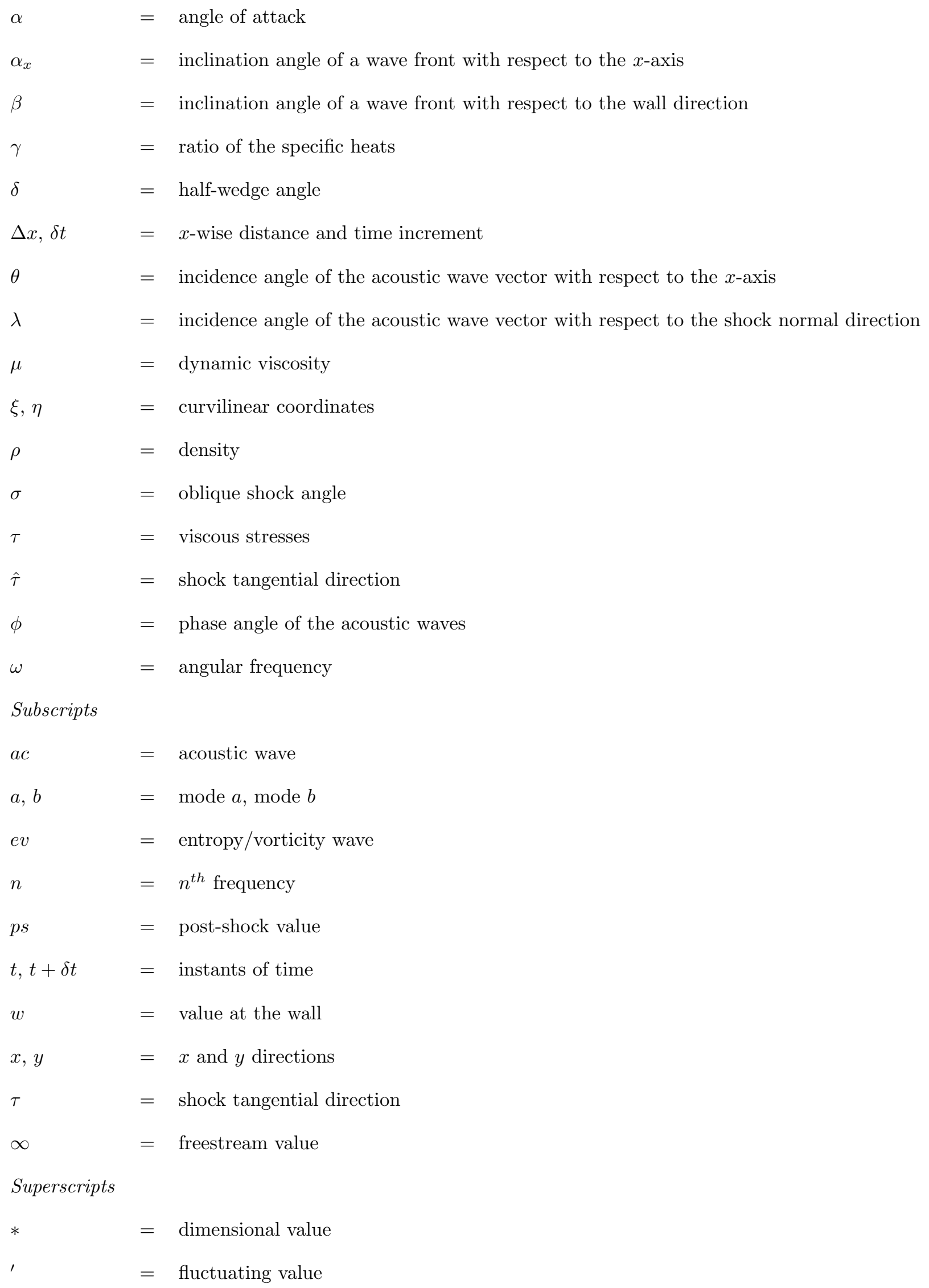




\section{Introduction}

The prediction of laminar-turbulent transition is a key factor in the aero-thermal design of hypersonic vehicles. As transition has a dramatic effect on skin friction and heat transfer rates on the surface of a body flying at hypersonic speeds, it is necessary to understand the main mechanisms leading to transition in hypersonic boundary layers, in particular in order to accurately predict the transition point. The main route from the laminar to turbulent state, in the case of small amplitude environmental disturbances, can be summarized in the following three fundamental processes: a) receptivity, b) eigenmode growth of the instabilities and c) nonlinear instability and breakdown to turbulence. Receptivity is the process by which external disturbances enter the boundary-layer and are converted into internal boundary-layer waves travelling downstream. The initial amplitudes, frequencies and phase velocities of these waves depend on the characteristics of the receptivity process, and represent initial conditions for the downstream growth of boundary-layer instabilities up to the nonlinear breakdown. The second stage consists of the propagation of small-amplitude instability waves downstream, corresponding to the boundary-layer linear stability problem. When the amplitudes of the instability waves reach significant values, the flow enters the last stage of the path to transition, where nonlinear effects take place, causing breakdown and final transition into a turbulent state.

In the present paper we are interested in the leading-edge receptivity of hypersonic boundary layers over a $2 \mathrm{D}$ cylinder-wedge geometry, whose sketch is presented in figure 1.

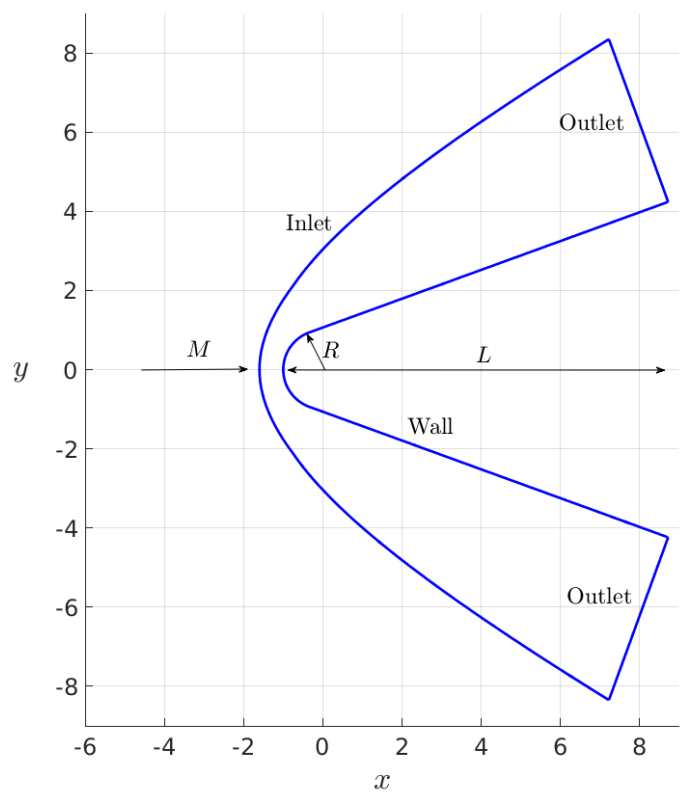

Figure 1: Sketch of the computational domain in the nose region. $R$ and $L$ denotes the nose radius and the length respectively. 
The Mach number plays a crucial role in the receptivity process, providing important differences between the subsonic regime and the supersonic and hypersonic regimes. In particular, in contrast to the subsonic regime, where the receptivity is linked to a wavelength-conversion mechanism, as described by Saric et al [1], at supersonic Mach numbers the generation of the boundary-layer modes is essentially due to a direct resonant interaction between the internal waves and the freestream disturbances, as shown in several theoretical and numerical studies (Fedorov and Khokhlov[2], Fedorov[3, Balakumar[4, Zhong and Ma [5], Zhong and Wang[6]). Moreover, while the characteristic unstable mode in the subsonic and low-supersonic flow regimes is represented by the first instability mode (i.e. the Tollmien-Schlichting waves), in the high-supersonic to hypersonic regime other higher frequency so-called Mack modes can coexist in the boundary layer, as shown by Mack [7], among which the second mode was observed in Stetson's experiment [8] to be the dominant boundary-layer mode in the transition process.

Another distinction needs to be made, concerning the type of environmental disturbances interacting with the boundary layer. In general, the environmental noise of a hypersonic wind tunnel can be considered as composed by all the types of waves, namely fast and slow acoustic waves, entropy waves, and vorticity waves. This increases the degree of complexity of the disturbance field in a hypersonic wind tunnel, which is, in turn, very difficult to measure (Schneider [9]). In a recent numerical study, Duan et al. [10] showed that the noise generated by a fully turbulent boundary layer in a flow at Mach 2.5 over a flat plate is mainly characterized by acoustic disturbances with wave-front orientation and phase speed belonging to the class of slow acoustic waves. This is an indication that slow acoustic modes are efficiently produced by turbulent boundary layers on the wind tunnel walls.

Furthermore, the characteristics of the waves interacting with the boundary layer depend on the shockdisturbance interaction. Ma and Zhong [11] numerically studied the receptivity of a Mach 4.5 flow over a flat plate to different types of freestream disturbances, and observed that, in the case of freestream entropy and vorticity waves, the boundary-layer disturbances are mainly induced by fast acoustic waves generated behind the shock by the freestream forcing waves.

Considering the interaction of different types of wave with the boundary layer, the theoretical studies by Fedorov and Khokhlov[2, and Fedorov[3] showed that fast acoustic, entropy and vorticity waves produce a similar receptivity mechanism, which consists of the generation and early amplification, due to a direct resonance (or synchronization) mechanism with the forcing waves at the leading edge, of a stable internal mode (mode F, or fast mode), which decays further downstream, while for slow acoustic waves the resonance mechanism at the leading edge produce the direct generation of mode $\mathrm{S}$ (or slow mode), which becomes unstable and grows downstream of the neutral point. The receptivity mechanism to fast and slow acoustic waves has been investigated through direct numerical simulations (DNS) by several authors, e.g. Zhong and 
Ma [5], Balakumar 4, Kara et al.[12, Malik and Balakumar[13], Egorov et al.[14].

The receptivity mechanism in the upstream region, namely the region extending from the leading edge to the lower neutral point of the second instability mode, is of fundamental importance for the growth mechanism of the instabilities downstream in the boundary layer. The aim of the present work is to study the leading-edge receptivity mechanism to fast and slow freestream acoustic waves for six different supersonic/hypersonic flow conditions over a wedge-shaped geometry, with a focus on the characteristics of the wave structure induced in the region downstream of the shock by the different freestream wave types, and on the generation and evolution of the boundary-layer disturbances along the wall in the upstream region. The response is analyzed in terms of the wall pressure fluctuation amplitudes, and the influence of the Mach number, angle of attack and inclination angle of the acoustic waves with respect to the flow direction, on both the post-shock wave structure and the wall response, is evaluated. These numerical simulations cover a set of experiments carried out at DLR (Wagner et al. [15]), aimed at linking the pre-transitional surface pressure fluctuations measured over a wedge probe to the freestream disturbances in hypersonic wind tunnels.

\section{Numerical method}

\section{II.A. Governing equations}

We consider numerical solutions of the three-dimensional Navier-Stokes equations for compressible flows, under the assumption of a perfect gas. The current simulations are $2 \mathrm{D}$ since it is believed that the dominant unstable mode in two-dimensional hypersonic boundary layers in the linear regime is the $2 \mathrm{D}$ second instability mode (as shown in the works of Fedorov [3] and Zhong and Wang [6]). A separate study considering the effects of 3D oblique acoustic waves on the receptivity and breakdown mechanisms in hypersonic boundary layers has been presented in Cerminara and Sandham [17. For the two-dimensionality of our numerical simulations, the set of non-dimensional conservation equations in curvilinear coordinates can be written in conservation form as

$$
\frac{\partial \mathbf{Q}}{\partial t}+\frac{\partial \mathbf{F}}{\partial \xi}+\frac{\partial \mathbf{G}}{\partial \eta}=0
$$

where $(\xi, \eta)$ are the curvilinear coordinates, while the Cartesian coordinates are $x=x(\xi, \eta)$ and $y=y(\xi, \eta)$.

6 of 27

American Institute of Aeronautics and Astronautics 
In the equation above, $\mathbf{Q}$ is the vector of the conservative variables, while $\mathbf{F}$ and $\mathbf{G}$ are the vectors of the fluxes in curvilinear coordinates. These vectors are linked to the corresponding vectors in Cartesian coordinates, namely $\mathbf{Q}_{c}, \mathbf{F}_{c}, \mathbf{G}_{c}$, by

$$
\mathbf{Q}=J \mathbf{Q}_{c}, \mathbf{F}=J\left(\mathbf{F}_{c} \frac{\partial \xi}{\partial x}+\mathbf{G}_{c} \frac{\partial \xi}{\partial y}\right), \mathbf{G}=J\left(\mathbf{F}_{c} \frac{\partial \eta}{\partial x}+\mathbf{G}_{c} \frac{\partial \eta}{\partial y}\right)
$$

where $J=\operatorname{det}\|\partial(x, y) / \partial(\xi, \eta)\|$ is the determinant of the Jacobian transformation matrix. The components of the vectors of the system in conservative form and in Cartesian coordinates are

$$
\mathbf{Q}_{c}=\left[\begin{array}{c}
\rho \\
\rho u \\
\rho v \\
\rho E
\end{array}\right] \quad \mathbf{F}_{c}=\left[\begin{array}{c}
\rho u \\
\rho u^{2}+p-\frac{1}{R e} \tau_{x x} \\
\rho u v-\frac{1}{R e} \tau_{x y} \\
\rho u\left(E+\frac{p}{\rho}\right)-\frac{1}{R e}\left(u \tau_{x x}+v \tau_{x y}+\frac{\mu}{(\gamma-1) \operatorname{Pr} M^{2}} \frac{\partial T}{\partial x}\right)
\end{array}\right]
$$

and

$$
\mathbf{G}_{c}=\left[\begin{array}{c}
\rho v \\
\rho u v-\frac{1}{R e} \tau_{x y} \\
\rho v^{2}+p-\frac{1}{R e} \tau_{y y} \\
\rho v\left(E+\frac{p}{\rho}\right)-\frac{1}{R e}\left(u \tau_{x y}+v \tau_{y y}+\frac{\mu}{(\gamma-1) \operatorname{Pr} M^{2}} \frac{\partial T}{\partial y}\right)
\end{array}\right]
$$

The terms $\rho, \rho u, \rho v$ and $\rho E$ are the conservative variables of the system of equations, where $\rho$ is the density, $u$ and $v$ are the velocity components respectively in the $x$, and $y$ directions, and $E$ is the total energy per unit mass. In the flux vectors, the terms $p, T, \tau_{x x}, \tau_{x y}, \tau_{y y}$, and $\mu$ are respectively the pressure, the temperature, the components of the viscous stress tensor, and the dynamic viscosity of the flow. The non-dimensional quantities are obtained through normalization of the dimensional variables with their freestream reference values: velocity components are normalized with the dimensional freestream main velocity $\left(U_{\infty}^{*}\right)$, density is normalized with the freestream density $\left(\rho_{\infty}^{*}\right)$, viscosity with the freestream dynamic viscosity $\left(\mu_{\infty}^{*}\right)$, temper- 
ature with the freestream temperature $\left(T_{\infty}^{*}\right)$, total energy with the square of the freestream main velocity $\left(U_{\infty}^{* 2}\right)$, while the pressure and viscous stresses are normalized with the term $\rho_{\infty}^{*} U_{\infty}^{* 2}$, related to the freestream dynamic pressure. The nose radius $\left(R^{*}\right)$ is chosen as the characteristic length to normalize the length scales, while the time scales are normalized with respect to the fluid dynamic characteristic time $\left(R^{*} / U_{\infty}^{*}\right)$, based on the velocity of the undisturbed flow and the characteristic length. The terms $\operatorname{Re}, \operatorname{Pr}, M$, and $\gamma$ are respectively the Reynolds, Prandtl and Mach numbers, and the ratio of specific heats $\left(\gamma=c_{p} / c_{v}\right)$, i.e. the dimensionless parameters of the flow. The Reynolds number is defined with respect to the nose radius, as $R e=\left(\rho_{\infty}^{*} U_{\infty}^{*} R^{*}\right) / \mu_{\infty}^{*}$; the Prandtl number is set to 0.72 for air, and $\gamma$ is equal to 1.4. The dynamic viscosity is expressed in terms of temperature by Sutherland's law

$$
\mu=T^{3 / 2} \frac{1+C}{T+C}
$$

where the constant $C$ represents the ratio between the Sutherland's constant (set to $110.4 \mathrm{~K}$ ) and the reference temperature $\left(T_{\infty}^{*}\right)$. The viscous stresses are defined in terms of the velocity derivatives, under the assumption of a Newtonian fluid, as

$$
\tau_{x x}=\mu\left[2 \frac{\partial u}{\partial x}-\frac{2}{3}\left(\frac{\partial u}{\partial x}+\frac{\partial v}{\partial y}\right)\right], \tau_{x y}=\mu\left(\frac{\partial u}{\partial y}+\frac{\partial v}{\partial x}\right), \tau_{y y}=\mu\left[2 \frac{\partial v}{\partial y}-\frac{2}{3}\left(\frac{\partial u}{\partial x}+\frac{\partial v}{\partial y}\right)\right]
$$

We also need a relation linking the total energy to the temperature, which in non-dimensional form can be expressed as

$$
E=\frac{T}{\gamma(\gamma-1) M^{2}}+\frac{1}{2}\left(u^{2}+v^{2}\right)
$$

Finally, the system of equations is closed by the equation of state for a perfect gas 


$$
p=\frac{1}{\gamma M^{2}} \rho T
$$

A fixed boundary condition is adopted at the inlet, with all the variables equal to their freestream values. After convergence to the steady-state flow, a time-periodic boundary condition is superimposed at the inflow for the unsteady simulations, with the variables varying in space and time according to an acoustic wave function (detailed in the following section). On the body surface an isothermal wall boundary condition is used, with wall temperature (normalised with respect to the freestream temperature) fixed at a value dependent on the particular case.

\section{II.B. Modeling of planar acoustic waves}

The freestream perturbation amplitudes of the physical quantities are expressed relative to the density perturbation amplitude $\left(\left|\rho^{\prime}\right|\right)$ by means of the following relations, derived from the linearized Euler equations under the assumption of small perturbations

$$
\left|u^{\prime}\right|=\frac{1}{M}\left|\rho^{\prime}\right| \cos \theta,\left|v^{\prime}\right|=\frac{1}{M}\left|\rho^{\prime}\right| \sin \theta,\left|p^{\prime}\right|=\frac{1}{M^{2}}\left|\rho^{\prime}\right|,\left|E^{\prime}\right|=\frac{1}{M}\left|\rho^{\prime}\right|\left(\frac{1}{\gamma M}+\cos \alpha \cos \theta+\sin \alpha \sin \theta\right)
$$

where $\alpha$ denotes the angle of attack, and $\theta$ the inclination angle of the acoustic waves with respect to the $x$-axis, considered positive for waves pointing upwards. The relations for the fluctuation amplitude of the velocity components and pressure are consistent with the dispersion relations shown in the work of Egorov et al.[14]; while the derivation of the total energy perturbation amplitude can be found in the work of Cerminara and Sandham [16].

Once the amplitude is assigned, the freestream perturbation of the density as a function of time and the Cartesian coordinates, for the case of multiple frequencies, is expressed as

$$
\rho^{\prime}(x, y, t)=\left|\rho^{\prime}\right| \sum_{n=1}^{N} \cos \left(k_{n x} x+k_{n y} y-\omega_{n} t+\phi_{n}\right)
$$


where $k_{n x}$ and $k_{n y}$ are the wavenumbers respectively in the $x$ and $y$ directions, $\omega_{n}$ is the angular frequency and $\phi_{n}$ the phase angle of the acoustic wave for the $n^{t h}$ frequency, and $N$ represents the total number of frequencies of the wave spectrum. These terms are, in turn, expressed by

$$
k_{n x}=\left|k_{n}\right| \cos \theta ; k_{n y}=\left|k_{n}\right| \sin \theta ;\left|k_{n}\right|=\frac{\omega_{n}}{\cos \theta \pm 1 / M} ; \omega_{n}=n \omega_{1}=2 \pi n f_{1} .
$$

Here, $\left|k_{n}\right|$ is the magnitude of the wave vector for the $n^{\text {th }}$ frequency, for which the $+(-)$ sign applies for fast (slow) acoustic waves, and $f_{1}$ is the smallest frequency of the complete spectrum.

The perturbations of the other variables are easily obtained from the density perturbation function and the relations for the amplitudes listed above; in this way, the vector of the conservative variables at the inflow boundary in the unsteady computations is given by

$$
\mathbf{Q}_{c}^{U}=\left[\begin{array}{c}
\rho_{\infty}+\rho^{\prime} \\
\left(\rho_{\infty}+\rho^{\prime}\right)\left(u_{\infty}+u^{\prime}\right) \\
\left(\rho_{\infty}+\rho^{\prime}\right)\left(v_{\infty}+v^{\prime}\right) \\
\left(\rho_{\infty}+\rho^{\prime}\right)\left(E_{\infty}+E^{\prime}\right)
\end{array}\right]
$$

where the infinity symbol $(\infty)$ denotes mean values of the physical quantities in the freestream.

\section{II.C. Code features}

The code we use to carry out our DNS computations is the SBLI (Shock-Boundary-Layer-Interaction) code, developed over a number of years at the University of Southampton. The base scheme consists of a fourthorder central-differencing entropy-splitting method, in conjunction with a second-order Harten-Yee TVDtype (Yee et al.[18]) scheme used as a filter step for shock capturing. At the wall boundary a fourth-order Carpenter scheme (Carpenter et al.[19]) is used. For time integration, a third-order Runge-Kutta scheme is used. Moreover, a Ducros sensor (Ducros et al. 20]) is used in order to minimize the artificial dissipation in the vortical regions, in particular inside the boundary layer. The code works in parallel, using MPI libraries. A validation of the code can be found in the work of De Tullio et al.21. A grid study relevant for the current problem is presented in Section IV.A of this paper. 


\section{Flow conditions for the six cases and preliminary results}

Table 1 shows the flow conditions for the numerical cases studied. These represent the same flow conditions of a set of experiments carried out at DLR on a probe (Wagner et al. [15]) designed to measure the disturbance levels in high and low enthalpy hypersonic wind tunnels, through a combined approach between the surface pressure measurements and the numerical results for the wall-to-freestream pressure disturbances. In particular, cases 1 to 3 are relative to the tests in the Gottingen High Enthalpy Shock Tunnel (HEG), and cases 4 to 6 are relative to the tests performed in the DNW Ludwieg Tube (RWG). The nose radius of the probe geometry is $R^{*}=0.1 \mathrm{~mm}$, and the half-wedge angle is $\delta=20^{\circ}$. The simulations have been carried out under the assumption of perfect gas, which applies, for the present freestream conditions, to both the low enthalphy (RWG) and high enthalphy (HEG) cases, considering that, for example, the maximum temperature inside the boundary layer along the wedge is about $1020 \mathrm{~K}$ in case 1 (HEG, Mach 7.3, Re 440) and $950 \mathrm{~K}$ in case 2 (HEG, Mach 7.3, Re 140). Furthermore, the short duration of the tests prevents wall heating phenomena, and allows the wall temperature to be considered constant and equal to the room temperature.

Table 1: Flow conditions of the six numerical simulations

\begin{tabular}{rrrrrrrrr} 
Case & $M$ & $R e_{m}(1 / \mathrm{m})$ & $T_{0}^{*}(\mathrm{~K})$ & $T_{\infty}^{*}(\mathrm{~K})$ & $p_{\infty}^{*}(\mathrm{~Pa})$ & $T_{w}^{*} / T_{\infty}^{*}$ & $\alpha\left(^{\circ}\right)$ & $\theta\left(^{\circ}\right)$ \\
\hline 1 & 7.3 & $4.4 \times 10^{6}$ & 2740 & 234.034 & 2004.301 & 1.273 & 0 & 0 \\
2 & 7.3 & $1.4 \times 10^{6}$ & 2680 & 228.909 & 619.337 & 1.302 & 0 & 0 \\
3 & 7.3 & $1.4 \times 10^{6}$ & 2680 & 228.909 & 619.337 & 1.302 & 0 & 10 \\
4 & 6.0 & $6.3 \times 10^{6}$ & 559 & 68.571 & 588.852 & 4.346 & 0 & 0 \\
5 & 6.0 & $6.3 \times 10^{6}$ & 559 & 68.571 & 588.852 & 4.346 & 10 & 0 \\
6 & 3.0 & $12.0 \times 10^{6}$ & 258 & 92.538 & 3588.5 & 3.22 & 0 & 0
\end{tabular}

Preliminary simulations were carried out for case 1 with fast acoustic waves, over a small domain (grid size $360 \times 150)$ with streamwise length equal to 10 nose radii, and geometry presented in figure 1 , in order to check the linearity of the pressure response along the wall, with disturbance amplitudes ranging from $10^{-1}$ to $10^{-4}$. As shown in figure 1 the computational domain is made in such a way to be well adapted to both the body and the shock shape, based on the grid generation method by Bianchi et al 22 . The imposed single frequency in the preliminary simulations is high enough to provide a wavelength comparable with the size of the domain. Figure 2 shows the amplitude of the pressure fluctuations at different points along the wall versus the amplitude of the imposed density fluctuations in the freestream (from now on the amplitude symbol (| |) will be omitted). The essential linearity of the wall pressure response between the different freestream amplitudes at all the different points along the wall is shown. At all the different points the wall pressure fluctuation level scales linearly with the amplitude level of the freestream disturbances, 
which indicates that the response is linear within the considered amplitude levels. For all the cases listed in table 1 an amplitude of $1 \times 10^{-4}$ is chosen for the freestream density fluctuation, which, in view of the results shown in figure 2, is small enough to guarantee that the wall response is in the linear regime.

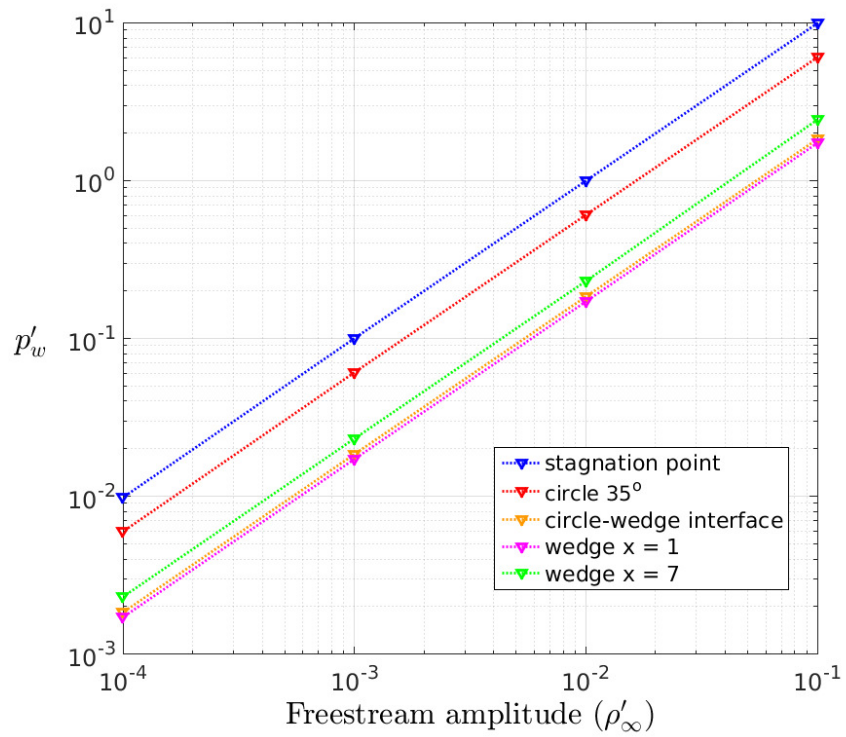

Figure 2: Pressure fluctuation amplitude at different points along the wall, from the stagnation point to the position $x=7$ (with the outlet at $x=9$ ), for increasing freestream amplitudes of the acoustic waves

\section{Unsteady simulations}

In this section we present numerical results for the cases listed in Table 1 with planar fast and slow acoustic waves, for multiple frequencies, random phase, and a density perturbation amplitude of $1 \times 10^{-4}$ at each frequency. The random phase has been added in order to avoid resonance sum of the wave peaks between the different frequencies, which would provide localised high-amplitude peaks along the freestream signal. The dimensionless frequency is normalized with the nose radius and the freestream velocity as $f=f^{*} R^{*} / U_{\infty}^{*}$. For all the fast-wave cases, a set of $N=10$ frequencies ranging from $f_{1}=50 \mathrm{kHz}$ to $f_{10}=500 \mathrm{kHz}$ has been imposed; while, for slow acoustic waves, 10 frequencies have been inserted from $f_{1}=20 \mathrm{kHz}$ to $f_{10}=200$ $\mathrm{kHz}$ for case 1 , from $f_{1}=25 \mathrm{kHz}$ to $f_{10}=250 \mathrm{kHz}$ for cases 2 and 3 , and from $50 \mathrm{kHz}$ to $500 \mathrm{kHz}$ for cases 4, 5 and 6 . These ranges were chosen in order to increase the frequency resolution at lower frequencies for the HEG cases (namely cases 1 to 3 ) with slow acoustic waves. The frequency range was chosen consistent 
with the known frequencies of the second instability mode, based on experimental observations (e.g., Estorf et al. [23]), and in agreement with the considered reference experiments (Wagner et al. [15]). The domain length is $400 R$ and the grid size for cases $1,2,3$ and 4 in Table 1 is $2244 \times 150$, while the grid size for cases 5 and 6 is $2244 \times 200$. For each simulation the steady state was obtained first, which required a number of iteration cycles on the order of $10000 R / U_{\infty}$, then the unsteady computation was run for about 10 periods (relative to the smallest frequency) of the acoustic waves to get periodic convergence. The Fourier analysis was performed over one cycle of the periodic disturbances.

\section{IV.A. Grid resolution study}

In figure 3 the results of a grid resolution study for one of the six numerical test cases $(M=6, R e=630$, $\alpha=0^{\circ}$, case 4) are presented, in which we compare the wall pressure fluctuation amplitude distributions at four selected frequencies (spanning the whole frequency range from the minimum, $50 \mathrm{kHz}$, to the maximum, $500 \mathrm{kHz}$, frequency with a step of $150 \mathrm{kHz}$ ) between two different grids. The grid we refer to as the fine grid is the reference grid size used to perform all our numerical simulations, namely $2244 \times 150$, while the coarser grid has been obtained by lowering the reference grid size by $1 / 3$ in both directions. The domain for the coarse grid is half of the domain used for the target numerical simulations, corresponding to a length in the streamwise direction of approximately 200 nose radii and to a grid size of $908 \times 100$ (considering a non-uniform point distribution along the streamwise direction, with higher density in the nose region).

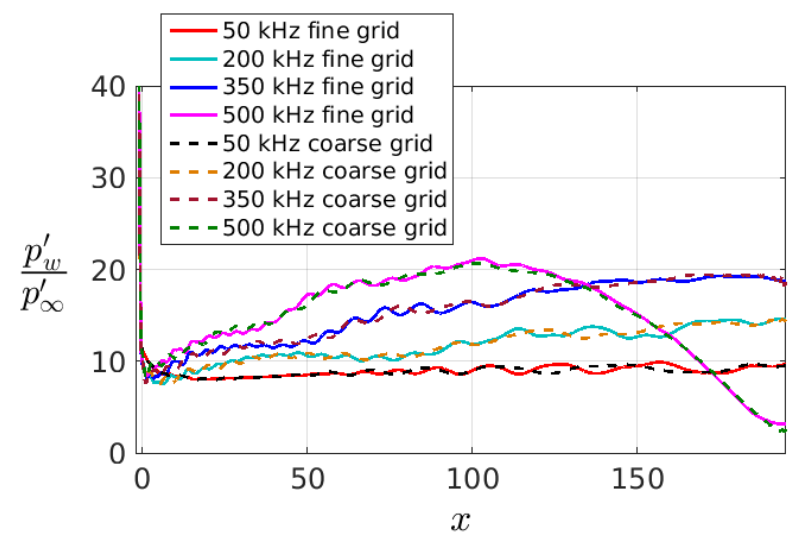

Figure 3: Wall pressure fluctuation amplitudes for two different grid levels, the fine grid (solid lines) and the coarse grid (dashed lines), at different frequencies. Case 4 , Mach $=6, \alpha=0^{\circ}$

As can be seen, the results of the grid resolution study for the pressure fluctuations along the wall show a very good agreeement between the two grids at all the considered frequencies, indicating that the solution can be considered as grid independent in the whole frequency range. The results show also the frequency effect on the wall response, which is seen to produce a higher early amplification of the fluctuation amplitude at increasing frequencies. The response at the maximum frequency $(500 \mathrm{kHz})$ shows that this frequency- 
dependent growth in the nose region reaches a peak at a certain distance from the leading edge, which is then followed by a decay further downstream. This shape of the wall response would, in general, also be shown by lower frequencies if a longer domain in the streamwise direction was considered. This behavior is due to a resonance mechanism at the leading edge between the external forcing waves and the induced boundary-layer modes, and will be discussed in more detail later.

\section{IV.B. Structure of the disturbance field}

The structure of the disturbance field behind the shock wave is analyzed through plots of the density fluctuation field for the Mach 3 case (case 6), shown in figures 4 and 5 for the fast and the slow acoustic wave case respectively. As can be seen, waves with significantly different orientation are formed downstream of the shock for fast and slow acoustic waves. Figure 6 shows a sketch of the inclination angles and phase speeds of the wave fronts upstream and downstream of the shock, for the fast-wave case, illustrating the characteristics of the propagation of the main waves in the post-shock region. A generic freestream wave front (depicted by a black dashed line aligned in the vertical direction) is labelled as $w_{t}$ and $w_{t+\delta t}$ at the instants of time $t$ and $t+\delta t$ respectively. The blue oblique dot-dashed line along the shock indicates the shock direction, and $\sigma$ the angle of the oblique shock with respect to the $x$-axis. The angle $\beta$ represents the inclination angle of the wave front in the post-shock region with respect to the wall direction, and $\delta=20^{\circ}$ is the half-wedge angle. The vectors in the $x$-direction denoted by $c_{\infty}$ and $c_{p s}$ represent respectively the freestream and the post-shock phase speed of the disturbances in the $x$-direction (the main propagation direction of the imposed waves), which for a time unit $\delta t=1$ are coincident with the $x$-displacement vectors of two different points on the same wave front upstream and downstream of the shock. The lower length of the vector $c_{p s}$ indicates that the portion of the wave front that has crossed the shock is propagating with a lower streamwise phase speed compared with the portion of the wave front still upstream of the shock, so that, after the time delay $\delta t$, the post-shock wave front reaches a point that is upstream of the point reached by the freestream wave front. These two points are located on the inclined black dashed line connecting the shock to the wall, which represents the deflected (or dragged) wave front due to the lower phase speed in the post-shock region. As the geometry is two-dimensional and the region of the computational domain considered in figure 6 is far enough away from the leading-edge (thus from the curvilinear part of the shock), the flowfield and the disturbance field behind the shock outside the boundary layer can be considered as approximately uniform, which explains why the post-shock dragged waves appear as oblique straight lines running from the shock to the boundary layer.

For the specific flow conditions of case 6 (Mach 3), the freestream phase speed is $c_{\infty}=u_{\infty}+a_{\infty}=$ $1+1 / M=1.33$. A theoretical estimation of the phase speed behind the shock, $c_{p s}$, can be made through 
inviscid oblique shock theory, which links the $x$-convection velocity $u_{p s}$ and the local speed of sound $a_{p s}$ to the normal (to the shock) freestream Mach number through the normal shock jump relations. From knowledge of the angle $\sigma$ of the oblique shock for a supersonic flow at Mach 3 over a wedge of $\delta=20^{\circ}$ halfwedge angle $\left(\sigma=37.7^{\circ}\right)$, the normal freestream Mach number can be computed as $M_{n}=M \sin \sigma=1.83$, and, by applying the shock jump relations, we compute a value for the post-shock $x$-wise phase speed equal to $c_{p s}=u_{p s}+a_{p s}=1.195$. From the values of $c_{\infty}$ and $c_{p s}$, the inclination angle of the wave front in the post-shock region with respect to the $x$-axis $\left(\alpha_{x}\right)$ can be computed through the relation

$$
\alpha_{x}=\tan ^{-1}\left(\frac{c_{\infty} \tan \sigma}{c_{\infty}-c_{p s}}\right)
$$

which gives a theoretical estimation of the wave front inclination angle of $\alpha_{x}=82.5^{\circ}$. This angle represents the sum of the angle $\beta$ (see figure 6) and the half-wedge angle. The numerical value of $\alpha_{x}$ corresponding to the results shown in figure 6 (i.e. inclination of the wave front in the post-shock region) is $\alpha_{x}=\beta+\delta \approx 81^{\circ}$, which is close to the theoretical estimation (with a relative error of $1.8 \%$ between the theoretical and the numerical results), indicating that the inclined wave fronts behind the shock in figure 6 are indeed dragged waves propagating with the velocity of the local fast acoustic waves behind the shock.

The presence of another type of wave with an opposite inclination can be noticed in figure 4 (whose direction is indicated by a red dashed line) close to the boundary layer, whose wave vector has an inclination angle of approximately $25^{\circ}$ with respect to the wall-tangential direction. As the local Mach angle at the boundary-layer edge is about $30^{\circ}$, this second type of wave appears to pertain to the class of fast waves reflected by the wall and propagating downstream with an angle close to the local Mach angle.

Case 6 with slow acoustic waves, depicted in figure 5 , shows a significantly different structure of the waves behind the shock, made of wave fronts deflected of about $\alpha_{x}=112^{\circ}$ with respect to the $x$-axis, namely oriented in the opposite direction compared to the fast-wave case. This indicates that the post-shock wave structure is dominated by transmitted waves travelling with a phase speed higher than the freestream phase speed of the slow acoustic waves, for example waves convected by the flow. Applying the same method used for the fast-wave case, but assuming now post-shock waves propagating in the local flow direction, we obtain an estimated value for the $x$-component of the post-shock phase speed of $c_{p s}=0.76$, which is very close to the numerical value of the local $x$-wise convection velocity component $u_{p s}=0.774$. As the numerical values of the local $x$-wise phase speeds of fast and slow acoustic waves are $c_{F p s}=u_{p s}+a_{p s}=1.193$ and $c_{S p s}=u_{p s}-a_{p s}=0.355$ respectively, we deduce that in this case the post-shock waves are convected waves (including vortical and entropy waves). 


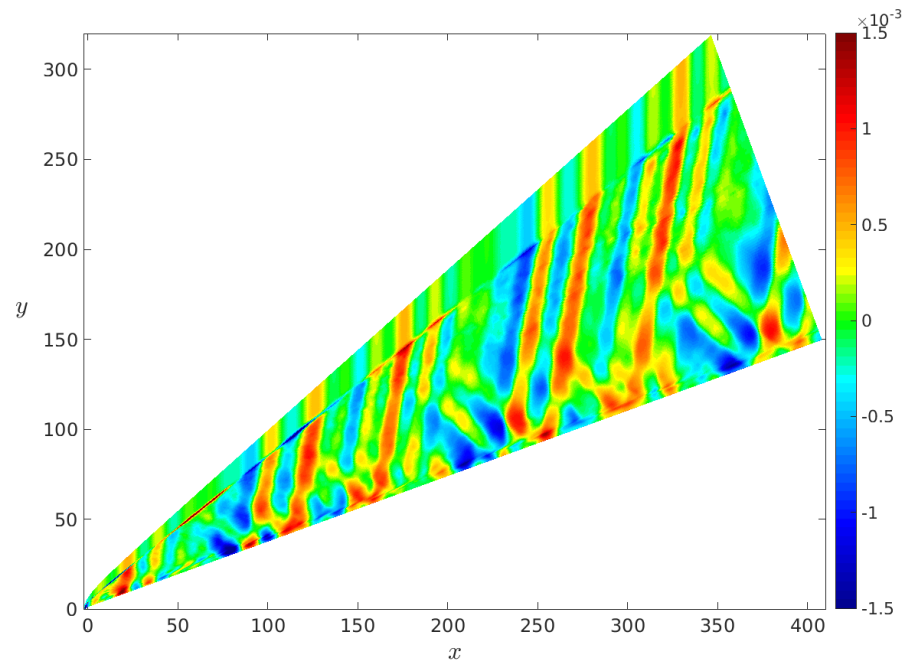

Figure 4: Density fluctuation field for fast acoustic waves. Considering the symmetry of the solution, only half the domain is shown. Case 6, Mach $=3$

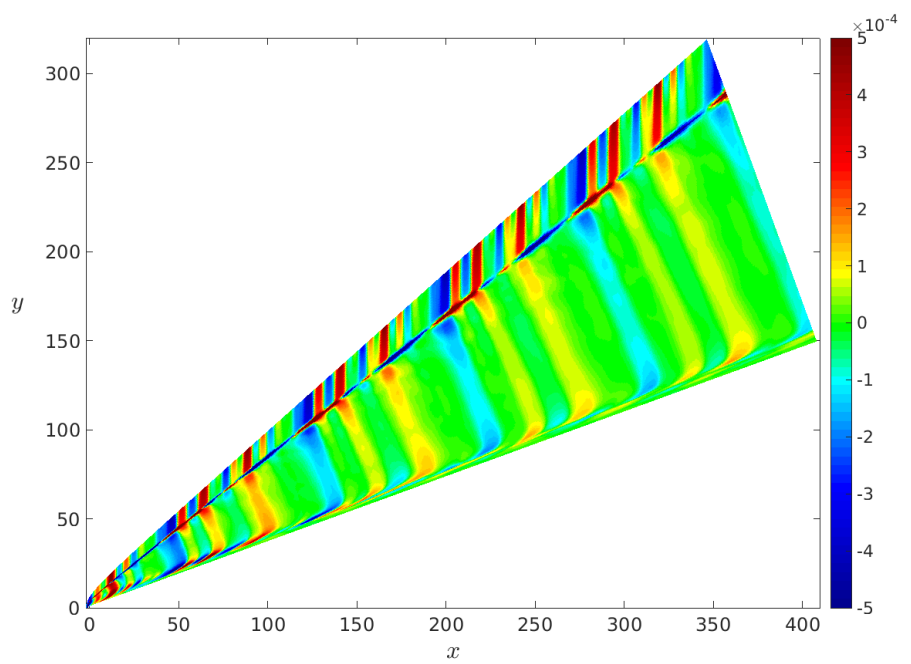

Figure 5: Density fluctuation field for slow acoustic waves. Considering the symmetry of the solution, only half the domain is shown. Case 6, Mach $=3$ 


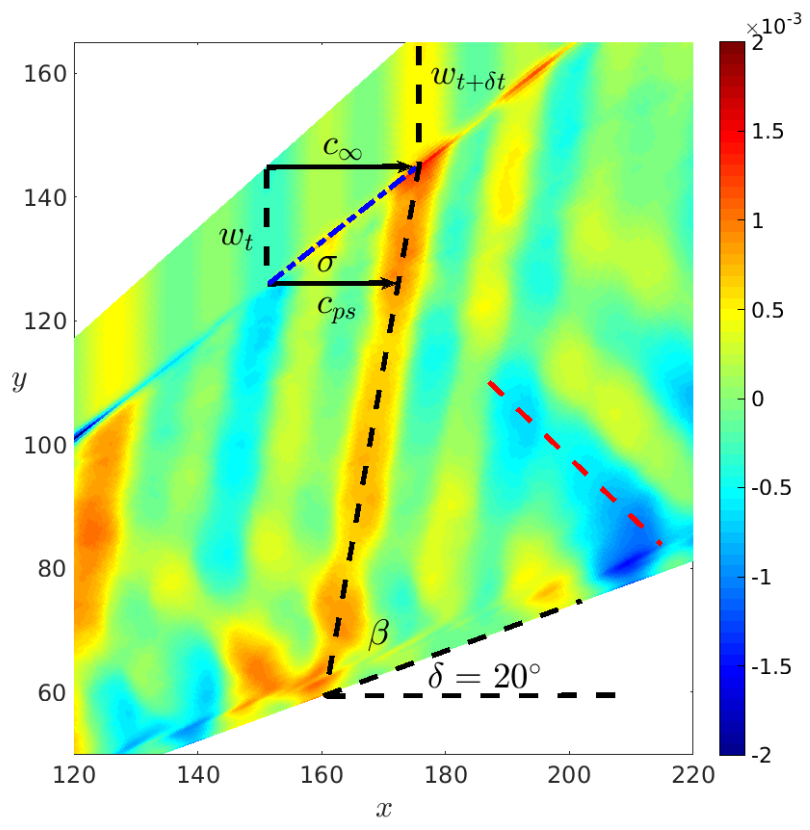

Figure 6: Expanded view of the density fluctuation field for fast acoustic waves, and sketch of the propagation features of the wave fronts upstream and downstream of the shock (Case 6 , Mach $=3$ )

\section{IV.B.1. Comparison with linear theory}

The structure of the disturbance field in the post-shock region (illustrated in section IV.B for the Mach 3 case) can be compared with the interaction theory of linear freestream disturbances and oblique shock waves, described by McKenzie and Westphal [24, who formulated Snell's laws of refraction for plane acoustic and non-acoustic waves, in the limit of small disturbances. Following this approach, an equation giving the inclination angle of the diverging wave in the case of an incident acoustic wave generating an acoustic wave downstream of the shock was derived [24] as

$$
\begin{gathered}
\cos \lambda_{p s, a c}=\frac{-\left(a_{p s} / a_{\infty}\right)^{2} \sin ^{2} \lambda_{\infty} M_{n, p s}+\left\{\Theta\left[\Theta-\left(a_{p s} / a_{\infty}\right)^{2} \sin ^{2} \lambda_{\infty}\left(1-M_{n, p s}^{2}\right)\right]\right\}^{1 / 2}}{\left(a_{p s} / a_{\infty}\right)^{2} \sin ^{2} \lambda_{\infty} M_{n, p s}^{2}+\Theta}, \\
\Theta=\left(1+M_{n, \infty} \cos \lambda_{\infty}\right)^{2},
\end{gathered}
$$

and in the case of an acoustic wave generating an entropy/vorticity wave downstream of the shock as

$$
\tan \lambda_{p s, e v}=\frac{\left(a_{p s} / a_{\infty}\right)^{2} M_{n, p s}\left( \pm \sin \lambda_{\infty}\right)}{1 \pm M_{n, \infty} \cos \lambda_{\infty}}
$$

where $\lambda$ is the inclination angle of the wave vector $(\mathbf{k})$ with respect to the shock-normal direction, as 
illustrated in figure 7, the subscripts $\infty$ and ${ }_{p s}$ indicate quantities in the freestream and in the post-shock region respectively, $M_{n}$ is the Mach number in the shock normal direction, and the subscripts $a c$ and $e v$ refer respectively to acoustic and entropy/vorticity waves generated downstream of the shock. Equation 14 applies to an incident fast acoustic wave generating a fast wave behind the shock, while in equation 16 the $+(-)$ sign applies to fast (slow) incident waves.

From the numerical oblique shock angle $\left(\sigma=38.5^{\circ}\right)$, the incidence angle of the freestream waves is $\lambda_{\infty}=90^{\circ}-38.5^{\circ}=51.5^{\circ}$, and by knowledge of the freestream normal Mach number and the speed of sound, and the corresponding numerical values downstream of the shock, equations 14,15 and 16 can be applied to our Mach 3 case. From equations 14 and 15 we obtain a refraction angle of the fast wave equal to $\lambda_{p s, a c}=41.04^{\circ}$, which is very close to the numerical refraction angle of $\lambda_{p s, a c}=\alpha_{x}-\sigma=42.5^{\circ}$ (with $\alpha_{x}=81^{\circ}$ ). This confirms that the transmitted waves behind the shock are refracted fast acoustic waves, as was previously deduced based on the length of the streamwise phase-speed vectors upstream and downstream of the shock (illustrated in figure 6). It should be noted that convected waves may also be present in general, as indicated by equation 16 , but are found to be negligible compared to the dominant refracted fast acoustic waves for the present case.

In the same way, applying equation 16 to the Mach 3 incident slow-wave case gives a theoretical refraction angle for the generated entropy/vorticity waves of $\lambda_{p s, e v}=74.53^{\circ}$, corresponding to a wave vector pointing upstream, which shows again a very good agreement with the numerical result of $\lambda_{p s, e v}=\alpha_{x}-\sigma=73.5^{\circ}$ (with $\alpha_{x}=112^{\circ}$ ). This confirms that the main waves generated behind the shock due to the interaction with incident freestream slow acoustic waves are indeed entropy/vorticity waves. In general there may also be refracted slow acoustic waves which manage to traverse the shock, but in this case they are swamped by the dominant convected waves.

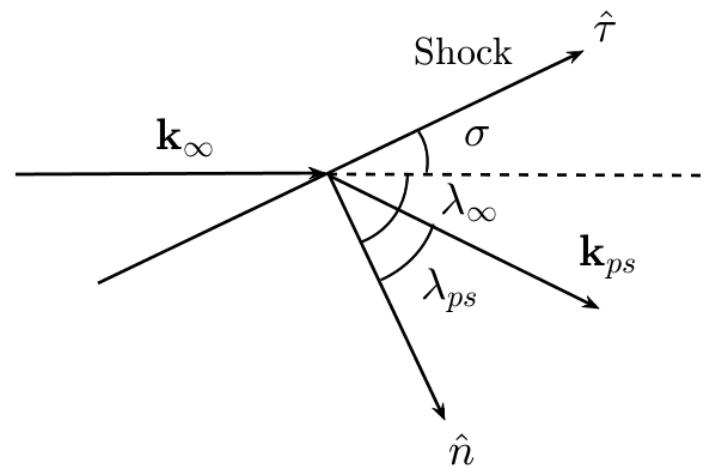

Figure 7: Sketch of the incidence and refraction wave angles in the shock-wave reference system. Here, $\hat{n}$ and $\hat{\tau}$ denote the shock normal and tangential directions respectively. 
Hence, the linear interaction theory is in agreement with our numerical results and, at the same time, validates the theoretical estimation method based on the relative length of the phase-speed vectors upstream and downstream of the shock.

\section{IV.C. Wall response: resonance and modulation behavior}

In this section the wall pressure fluctuations are analyzed for cases 2, 4 and 6 (see Table 1), in order to study the main characteristics of the leading-edge receptivity mechanism, namely the generation, interaction and downstream evolution of the induced boundary-layer modes, for different Mach numbers.

Figures 8a, 9a and 10a show the wall pressure fluctuation amplitudes at different frequencies for cases 2 , 4 and 6 respectively with fast acoustic waves, while figures $8 \mathrm{~b}$, 9b and $10 \mathrm{~b}$ show the corresponding results for slow acoustic waves. As can be seen, in case 2 the amplitudes gradually increase with distance from the leading edge for fast acoustic waves (figure 8a), while decreasing for the slow-wave cases (figure 8b), with a frequency-dependent growth/decay rate. This difference in the response to fast and slow acoustic waves is the effect of a stronger resonance mechanism for the fast waves, namely the synchronization between the forcing mode of the fast acoustic waves and the internal boundary-layer fast mode (mode F, according to Fedorov [3]'s notation) that is generated at the leading edge. This leads to an initial amplification of mode $\mathrm{F}$, in contrast to a weaker resonance mechanism in the case of slow acoustic waves, leading to the generation and initial decay of the boundary-layer slow mode (mode S). The difference in the resonance mechanisms is due to the characteristics of the wave transmission and propagation in the post-shock region, which determine the disturbance field forcing the boundary layer. As described in the previous section, in the fast-wave case the transmission of the freestream acoustic waves across the shock appears to be more efficient compared to the slow-wave case, with the main freestream wave fronts being deflected and amplified as they cross the shock. In the slow wave case, in contrast, the post-shock wave structure is dominated by lower amplitude convected waves, which are not synchronized with the slow mode generated at the leading edge. The interaction of the slow mode with the forcing waves of the post-shock region does not produce an efficient resonance mechanism, thus the response in the early nose region shows an initial decay.

A pronounced frequency-dependent oscillatory behavior of the wall response for the fast-wave case is seen in figures $9 \mathrm{a}$ and $9 \mathrm{~b}$, for case 4 with fast and slow waves respectively, and a substantially flat response (after an initial decay) for the slow-wave case. In this case, the wall response for both fast and slow waves appears as a continuation of the initial response shown by $8 \mathrm{a}$ and $8 \mathrm{~b}$ for case 2 . This is essentially because, as $U_{\infty}$

decreases with decreasing Mach numbers (for the cases listed in Table 1), the nondimensional frequencies increase, for the same dimensional frequency range.

The large-wavelength oscillations shown in the fast-wave case are due to a modulation process between 
the decaying mode $\mathrm{F}$ and the forcing acoustic mode, since after the initial synchronization the two are no longer coupled, as was observed in the numerical work of Zhong and Ma [5]. We can describe this modulation with a simplified model involving two different modes, the distance $(\Delta x)$ between two consecutive peaks of the oscillations, at a certain frequency $f$, is linked to the phase speeds of the two competing modes (modes $a$ and $b$ ) by (De Tullio and Sandham [25])

$$
\Delta x=\frac{c_{a} c_{b}}{f\left|c_{a}-c_{b}\right|}
$$

where $c_{a}$ and $c_{b}$ are the phase speeds of the two modes. Considering the wall response at $f=500 \mathrm{kHz}$ in figure 9a, the distance between two consecutive peaks downstream (located at $x=262.5$ and $x=372.5$ ) is $\Delta x=110$. Assuming $c_{a}$ to be coincident with the phase speed of the fast acoustic waves computed in the post-shock region $\left(c_{a}=c_{p s}=1.1\right)$, the phase speed of mode $b$, by applying equation 17 is $c_{b}=0.9179$. Hence, in the downstream region the mode of the forcing fast acoustic waves is modulated by a mode whose phase speed is lower than the fast-wave phase speed, and higher than the freestream slow-wave phase speed $(1-1 / M=0.83)$. This is consistent with the downstream gradual decay of mode $\mathrm{F}$, both in amplitude and phase speed. Hence, the two competing modes in the modulation region can be attributed to the forcing fast acoustic waves and the desynchronized boundary-layer mode F. This also means that the fast mode inside the boundary layer in the leading-edge region has an amplitude comparable with that of the external fast acoustic waves, which is possible due to the resonance mechanism.

This modulation behavior is even more evident in figure $10 \mathrm{a}$, for case 6 with fast acoustic waves. In contrast, figures $9 \mathrm{~b}$ and $10 \mathrm{~b}$, for cases 4 and 6 with slow acoustic waves, show at each frequency a faster initial decay, due to the absence of a strong resonance mechanism at the leading edge, followed by a flat overall response with a significantly lower amplitude compared to the fast-wave cases.

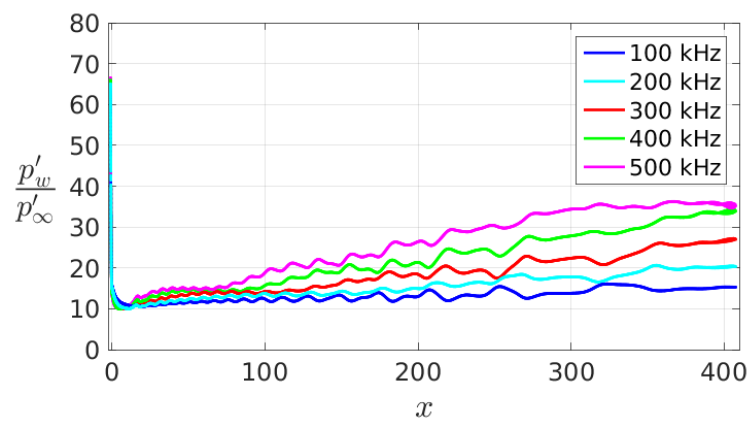

(a)

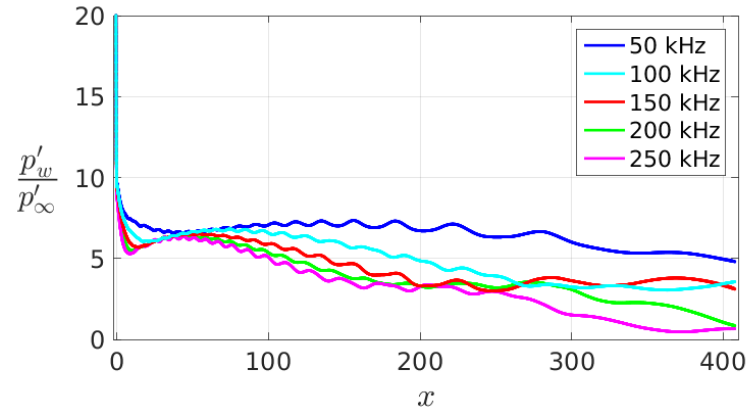

(b)

Figure 8: Wall pressure fluctuations for case 2 (Mach 7.3): (a) fast waves, (b) slow waves 


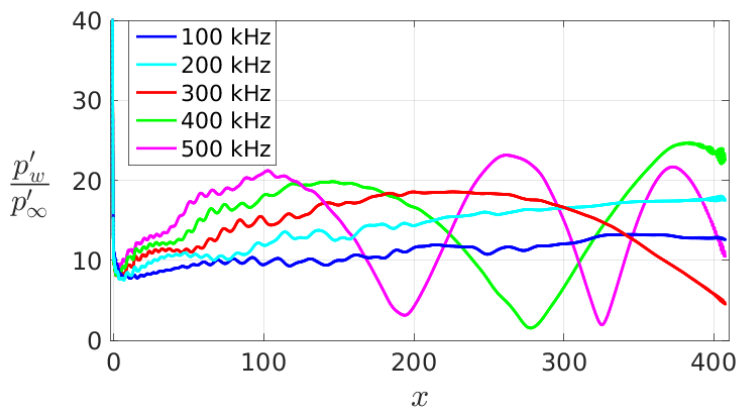

(a)

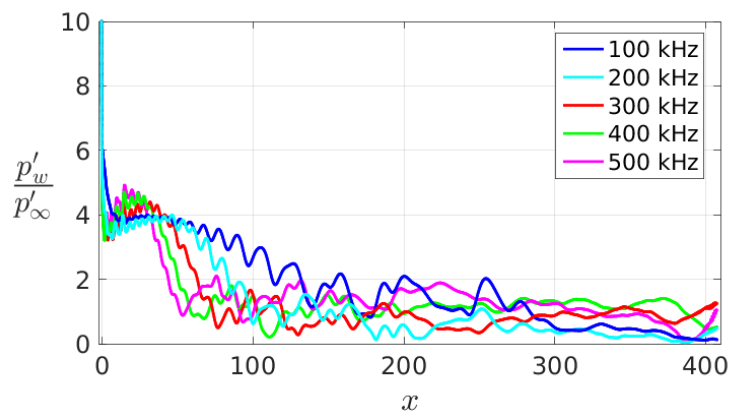

(b)

Figure 9: Wall pressure fluctuations for case 4 (Mach 6): (a) fast waves, (b) slow waves

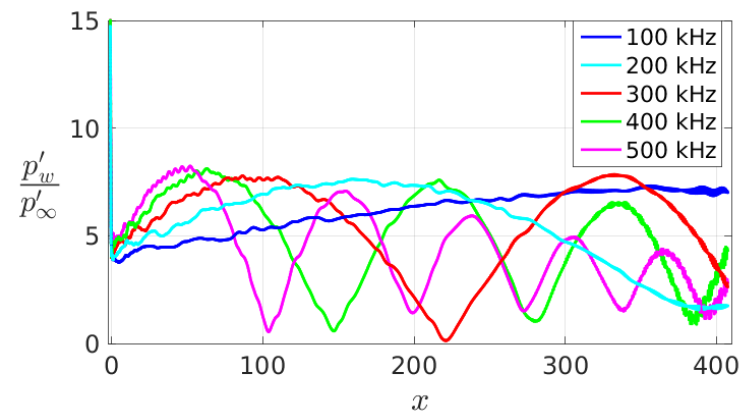

(a)

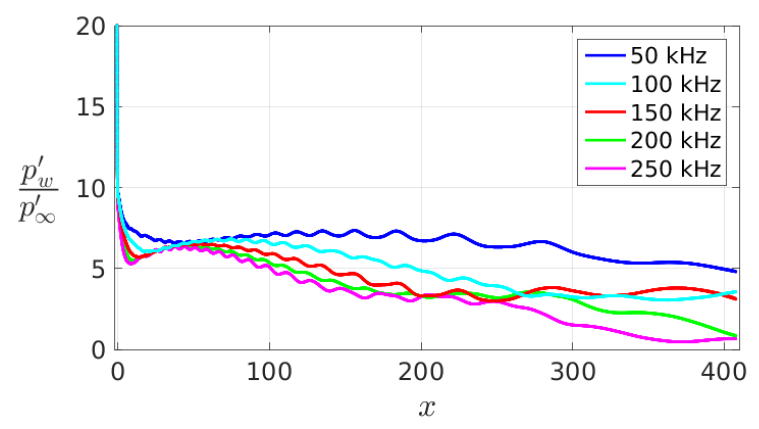

(b)

Figure 10: Wall pressure fluctuations for case 6 (Mach 3): (a) fast waves, (b) slow waves

\section{IV.D. Effect of wave inclination angle and angle of attack}

In this section we present results for the asymmetric cases 3 and 5, which show the effect of the angle of incidence of the acoustic waves (case 3) and the angle of attack (case 5) on the receptivity characteristics. With reference to Table 1 , in case 3 the fast and slow acoustic waves are introduced into the computational domain with an inclination angle $\theta=10^{\circ}$ with respect to the freestream (aligned with the $x$-axis). In case 5 , on the other hand, the wave vector of the acoustic waves is aligned with the freestream, which forms an angle of attack $\alpha=10^{\circ}$ with the symmetry axis of the geometry. In both cases 3 and 5 the bottom side of the wedge is the windward side, and the top side is the leeward side. Since for case 5 the flowfield is asymmetric due to the angle of attack, an asymmetric grid has been used, shaped in such a way to provide a good shock-grid alignment on both sides of the wedge.

Figures $11 \mathrm{a}$ and $11 \mathrm{~b}$ show the density fluctuation field for case 3 with fast and slow acoustic waves respectively, while figures $12 \mathrm{a}$ and $12 \mathrm{~b}$ show the corresponding results for case 5 . As can be seen, the results for case 3 show slight differences in the post-shock wave structure on the windward and lee sides for both fast and slow acoustic waves, which suggests a small effect of an incidence angle of $10^{\circ}$ on the receptivity 
patterns. In contrast, figures $12 \mathrm{a}$ and $12 \mathrm{~b}$ for case 5 reveal a significant effect of the angle of attack.

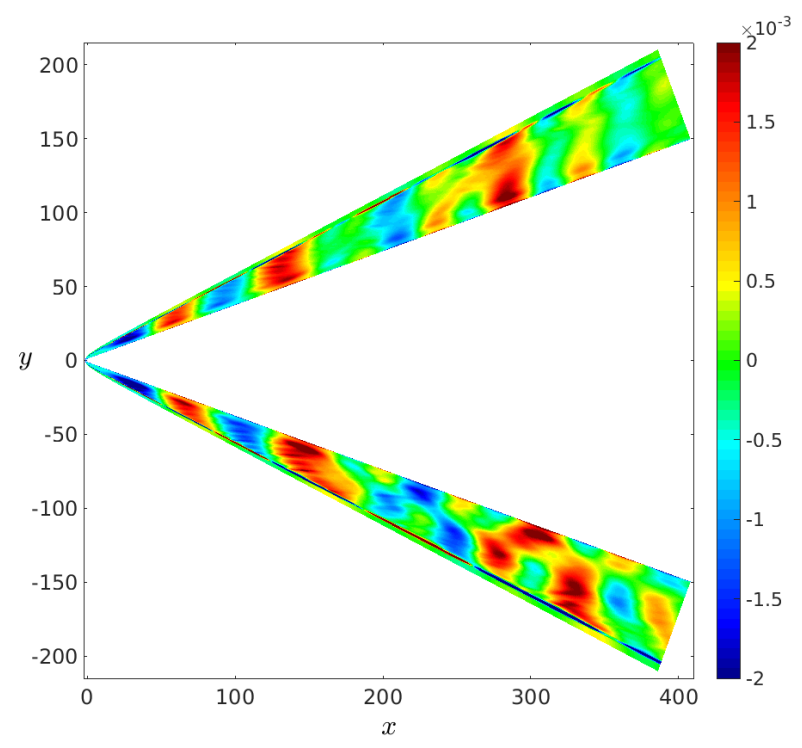

(a)

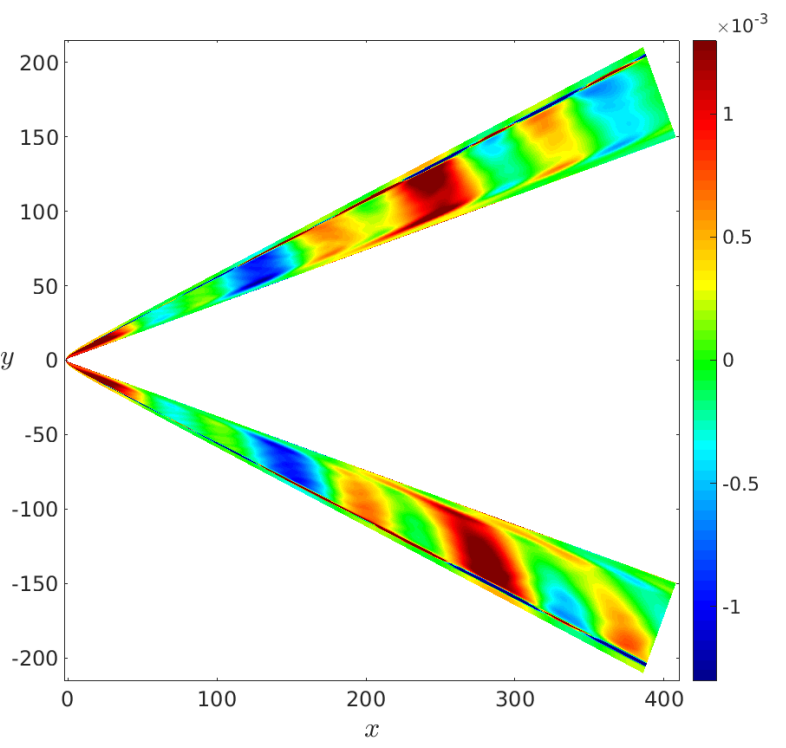

(b)

Figure 11: Density fluctuation field for case 3 (Mach 7.3): (a) fast waves, (b) slow waves

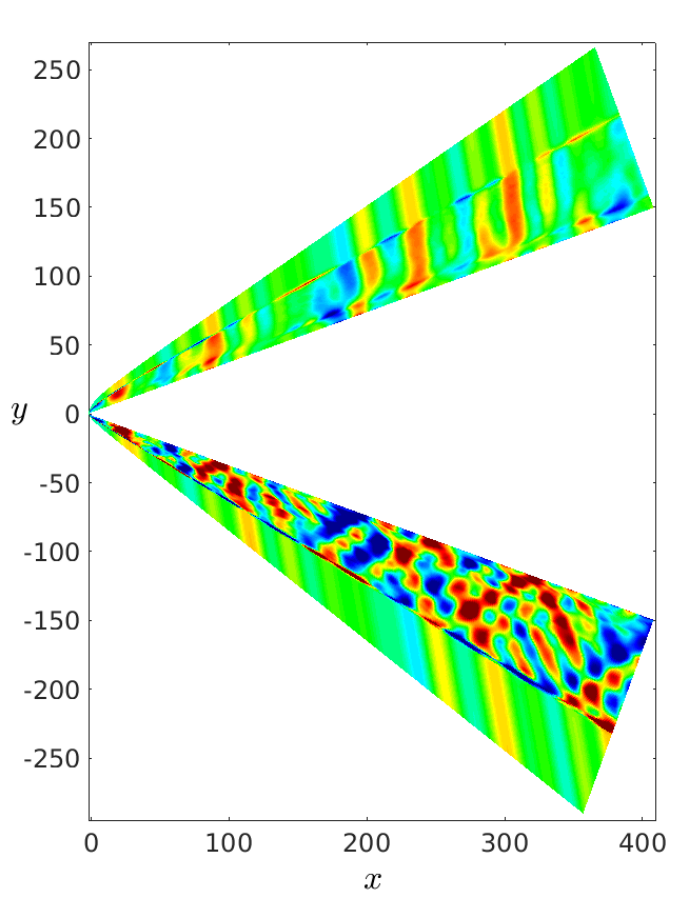

(a)

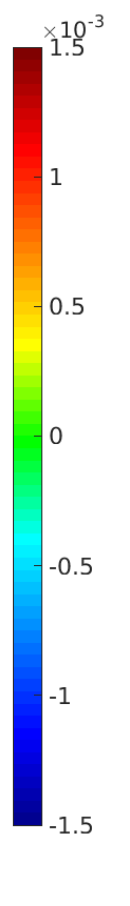

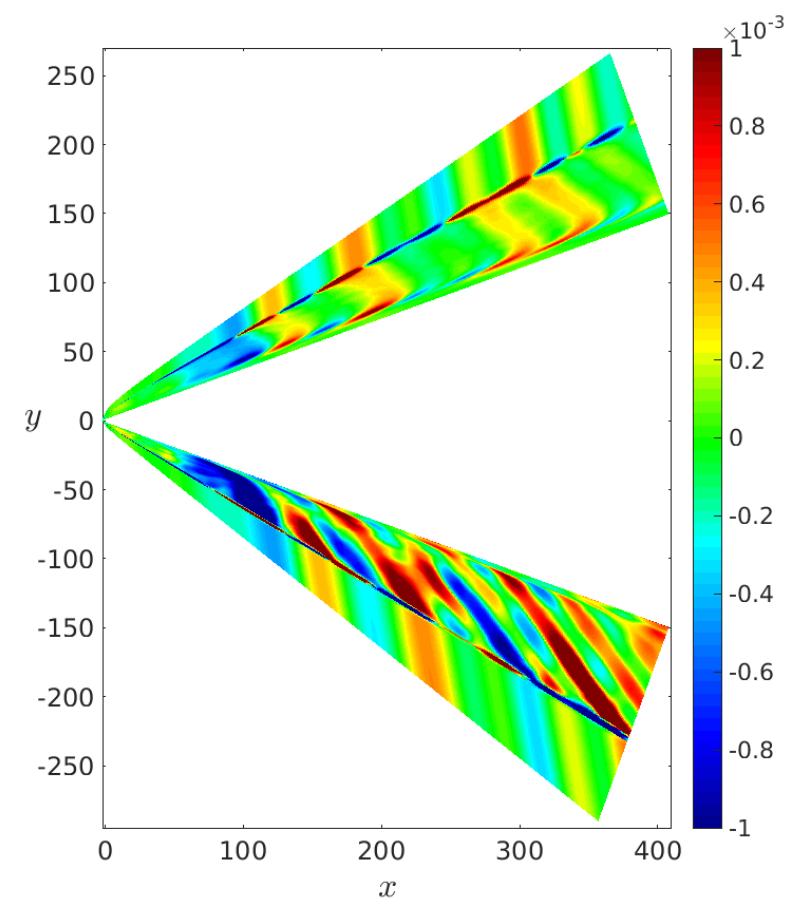

(b)

Figure 12: Density fluctuation field for case 5 (Mach 6): (a) fast waves, (b) slow waves 
The post-shock disturbances on the windward side (in figures $12 \mathrm{a}$ and $12 \mathrm{~b}$ have a significantly higher amplitude compared to the lee side, which is due to the shock being stronger on the windward side, which produces a higher disturbance amplification. The lee side shows the presence of dragged waves in the fast-wave case (figure 12a) and low-amplitude higher-phase-speed transmitted waves in the slow-wave case (figure 12b), as discussed in Section IV.B. In contrast, the windward side shows a complex system of dragged and reflected waves between the shock wave and the boundary layer for the fast-wave case, and a reversal (with respect to the lee side) of the post-shock phase speed in the slow-wave case, with the presence of high-amplitude dragged waves.

The different wave structures between the lee and the windward side have a strong effect on the boundarylayer receptivity characteristics, as is shown in figures $13 \mathrm{a}$ and $13 \mathrm{~b}$ for the wall pressure fluctuation amplitudes of case 3 , with fast and slow waves respectively, and similarly in figures $14 \mathrm{a}$ and $14 \mathrm{~b}$ for case 5 . In each plot the results are shown at two different frequencies and on both lee and windward sides. For case 3 with fast acoustic waves (figure 13a), the response at the lower frequency $(100 \mathrm{kHz})$ is very similar between the two wedge sides, being slightly higher on the windward side. At the higher frequency ( $500 \mathrm{kHz}$ ), the response is higher on the windward side in the nose region up to $x=250$, while dowstream of this point a reversal is observed, due to the response decay on the windward side and the downstream amplification along the lee side, in good agreement with the numerical results of Egorov et al. [14. An opposite trend is shown for the slow-wave case, whose results are presented figure 13b, thus indicating that the role of the wave incidence angle on the wall response is strongly dependent on the type of the incident waves.

The results for case 5 , presented in figures $14 \mathrm{a}$ and $14 \mathrm{~b}$ for fast and slow waves respectively, quantify the strong effect of the angle of attack on the wall receptivity, with the response on the windward side being significantly higher than on the lee side for both fast and slow acoustic waves.

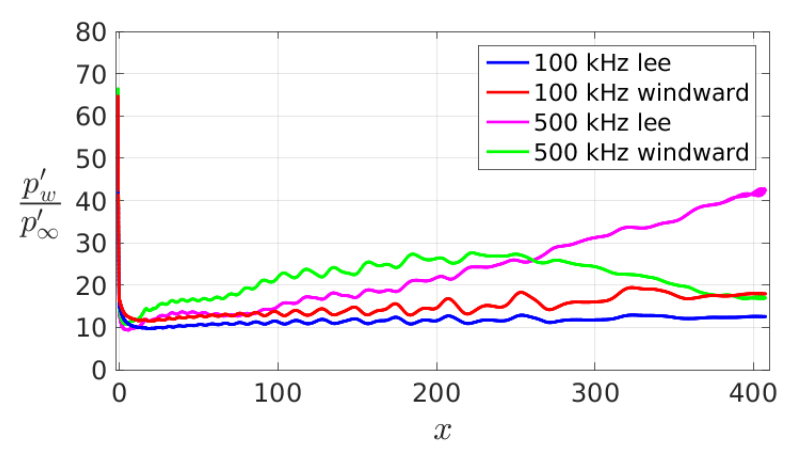

(a)

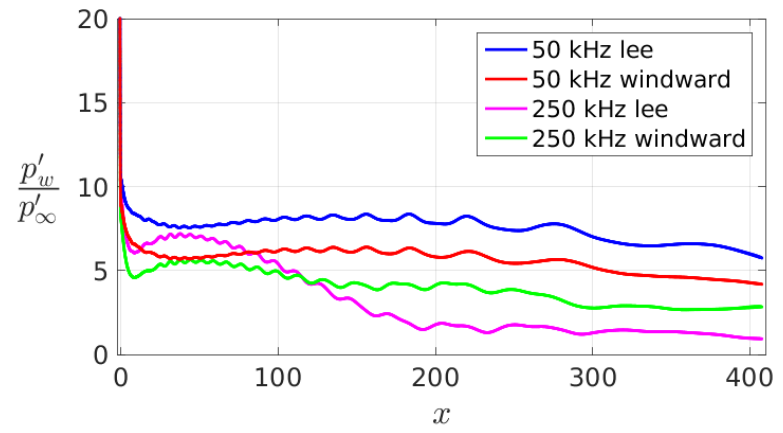

(b)

Figure 13: Wall pressure fluctuations for case 3 (Mach 7.3): (a) fast waves, (b) slow waves 


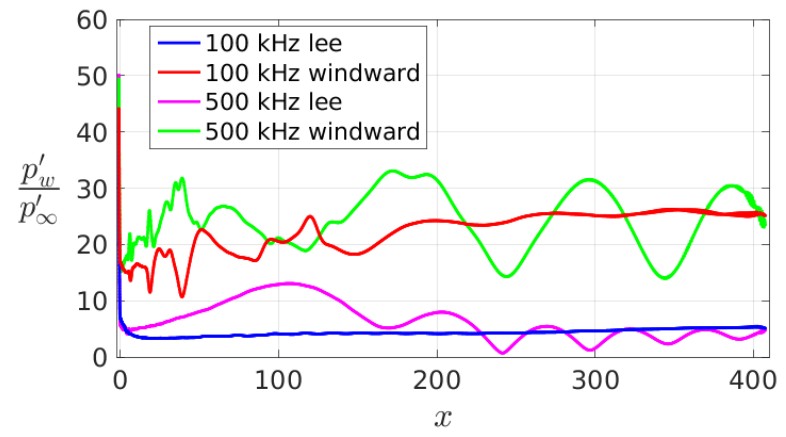

(a)

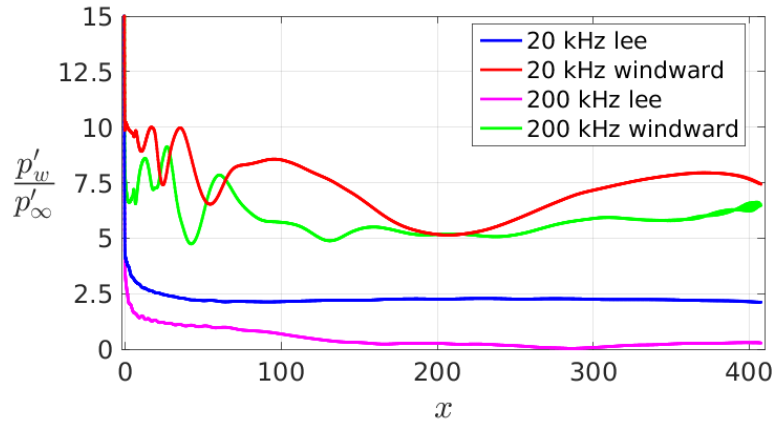

(b)

Figure 14: Wall pressure fluctuations for case 5 (Mach 6): (a) fast waves, (b) slow waves

\section{Conclusions}

The receptivity to fast and slow freestream acoustic waves with multiple frequencies at supersonic/hypersonic speeds over a 2D cylinder-wedge geometry, for a set of six flow conditions with different Mach number, Reynolds number, angle of attack, and angle of incidence of the acoustic waves, has been studied through DNS. The present study has focused on the immediate leading-edge region, which is characterized by the generation and interaction of the induced boundary-layer modes.

Three main aspects of the leading-edge receptivity to acoustic freestream disturbances in high speed flow have been analyzed, namely a) the structure of the disturbance field in the post-shock region, b) the response along the wall, with emphasis on the resonance-modulation mechanism, and c) the effect of an inclination angle of the acoustic waves and an angle of attack.

When planar fast acoustic waves are inserted into the freestream, the wave structure downstream of the shock is characterized by 'dragged' waves, pertaining to the main forcing fast acoustic waves traversing the shock and being deflected due to the lower local phase speed in the post-shock region. For freestream slow acoustic waves, in contrast, lower-amplitude higher-phase-speed waves are formed in the post-shock region, which have been shown to pertain to the class of local convected waves (namely entropy/vorticity waves) generated downstream of the shock from the interaction with the freestream slow waves.

Fast acoustic waves produce a higher response along the wall compared to slow waves, due to the different wave structure induced downstream of the shock and a stronger leading-edge resonance mechanism for the fast mode. This mechanism is seen to produce an early amplification of mode F until a first peak is reached, which is followed downstream by a frequency-dependent modulation behavior between the decaying mode $\mathrm{F}$ and the incident fast acoustic waves.

An angle of incidence of the fast acoustic waves of $10^{\circ}$ is seen to amplify the response along the lee side at 
the higher frequencies, while an opposite trend is seen for slow acoustic waves. An angle of attack of $10^{\circ}$ is shown to provide a significantly higher response along the windward side compared to the lee side, for both fast and slow acoustic waves, along with different characteristics of the post-shock wave structure between the two wedge sides.

This work has shown that the resonance mechanism at the leading edge for fast and slow waves is strongly dependent on the characteristics of the wave propagation downstream on the shock, including the type of the disturbances generated behind the shock from the interaction with the freestream waves. The efficiently-transmitted fast waves in the post-shock region trigger a powerful resonance mechanism at the leading edge for all the Mach numbers, while the conversion of the incident slow waves to weaker convected waves downstream of the shock inhibits the resonance. Thus the relative importance of fast and slow waves in the downstream evolution of the disturbances and the subsequent transition process depends on both the freestream conditions and the post-shock wave structure. In conclusion, the present work has identified the main mechanisms and parametric dependence of the leading-edge receptivity to acoustic disturbances in several high-speed flow conditions, and as such represents a basis for future investigations aimed at modeling the disturbance environment of conventional hypersonic wind tunnels, allowing experimental measurements on the surface of a wedge probe to be related to the freestream disturbances.

\section{Acknowledgments}

The authors would like to acknowledge MBDA UK Limited for supporting the PhD project of the first author. The work reported here formed part of an ESA/TRP project (contract number 22793/09/NL/CP). Computer time on the UK National Supercomputing Service (ARCHER) was provided by the UK Turbulence Consortium under EPSRC (Engineering and Physical Sciences Research Council) Grant No. EP/L000261/1. Data for the wall pressure, wall-tangential velocity and skin friction coefficient, and graphics of the temperature boundary-layer profiles, for the steady base flows, are available in the supplemental material (in the files named 'M7case1', 'M7case2', 'M6case4', 'M6case5', 'M3case6' and 'Tprofiles').

\section{References}

[1] Saric, William S., Helen L. Reed, and Edward J. Kerschen. Boundary-layer receptivity to freestream disturbances, Annual Review of Fluid Mechanics 34.1 (2002): 291-319. 
[2] Fedorov, Alexander V., and Andrew P. Khokhlov. Prehistory of instability in a hypersonic boundary layer, Theoretical and Computational Fluid Dynamics 14.6 (2001): 359-375.

[3] Fedorov, Alexander. Transition and stability of high-speed boundary layers, Annual Review of Fluid Mechanics 43 (2011): 79-95.

[4] Balakumar, P. Receptivity of a supersonic boundary layer to acoustic disturbances, AIAA journal 47.5 (2009): 1069-1078.

[5] Zhong, Xiaolin, and Yanbao Ma. Boundary-layer receptivity of Mach 7.99 flow over a blunt cone to free-stream acoustic waves, Journal of Fluid Mechanics 556 (2006): 55-103.

[6] Zhong, Xiaolin, and Xiaowen Wang. Direct numerical simulation on the receptivity, instability, and transition of hypersonic boundary layers, Annual Review of Fluid Mechanics 44 (2012): 527-561.

[7] Mack, Leslie M. Boundary-layer linear stability theory, California Institute of Technology, Pasadena Jet Propulsion Lab, 1984.

[8] Stetson, K. F., Thompson, E. R., Donaldson, J. C., and Siler, L. G. Laminar boundary layer stability experiments on a cone at Mach 8. II-Blunt cone, American Institute of Aeronautics and Astronautics, 22nd Aerospace Sciences Meeting, Reno, NV. 1984.

[9] Schneider, Steven P. Developing mechanism-based methods for estimating hypersonic boundary-layer transition in flight, The role of quiet tunnels." Progress in Aerospace Sciences 72 (2015): 17-29.

[10] Duan, Lian, Meelan M. Choudhari, and Minwei Wu. Numerical study of acoustic radiation due to a supersonic turbulent boundary layer, Journal of Fluid Mechanics 746 (2014): 165-192.

[11] Ma, Yanbao, and Xiaolin Zhong. Receptivity of a supersonic boundary layer over a flat plate. Part 3. Effects of different types of free-stream disturbances, Journal of Fluid Mechanics 532 (2005): 63-109.

[12] Kara, Kursat, Ponnampalam Balakumar, and O. A. Kandil. Receptivity of hypersonic boundary layers due to acoustic disturbances over blunt cone, Proceedings of the 37th AIAA Fluid Dynamics Conference and Exhibit, NASA Langley Research Center, 2007: 11464-11481.

[13] Malik, Mujeeb R., and Ponnampalam Balakumar. Acoustic receptivity of Mach 4.5 boundary layer with leading-edge bluntness, Theoretical and Computational Fluid Dynamics 21.5 (2007): 323-342.

[14] Egorov, I. V., V. G. Sudakov, and A. V. Fedorov. Numerical modeling of the receptivity of a supersonic boundary layer to acoustic disturbances, Fluid Dynamics 41.1 (2006): 37-48. 
[15] Wagner, Alexander, Erich Schuelein, and Klaus Hannemann. High Frequency Free-Stream Disturbance Measurements in Hypersonic Wind Tunnels by Means of a Slender Wedge Probe, AIAA Paper 2016-3192, 46th AIAA Fluid Dynamics Conference, Washington, D.C., 2016.

[16] Cerminara, Adriano, and Neil D. Sandham. Leading-Edge Receptivity to Acoustic Waves for High-Speed Flows over a Blunt Wedge, AIAA Paper 2015-3078, 45th AIAA Fluid Dynamics Conference, Dallas, TX, 2015.

[17] Cerminara, Adriano, and Neil D. Sandham. Boundary-layer receptivity and breakdown in hypersonic flow over a swept blunt wedge with three-dimensional freestream acoustic disturbances, AIAA Paper 2016-4247, 8th AIAA Flow Control Conference, Washington, D.C., 2016.

[18] Yee, H. C., Sandham, N. D., and Djomehri, M. J. Low-dissipative high-order shock-capturing methods using characteristic-based filters, Journal of Computational Physics 150 (1), 1999

[19] Carpenter, Mark H., Jan Nordstrm, and David Gottlieb. A stable and conservative interface treatment of arbitrary spatial accuracy, Journal of Computational Physics 148.2 (1999): 341-365.

[20] Yee, Helen C., Neil D. Sandham, and M. J. Djomehri. Low-dissipative high-order shock-capturing methods using characteristic-based filters, Journal of Computational Physics 150.1 (1999): 199-238.

[21] De Tullio, N., Paredes, P., Sandham, N. D., and Theofilis, V. (2013). Laminar-turbulent transition induced by a discrete roughness element in a supersonic boundary layer, Journal of Fluid Mechanics, 735 (2013): 613-646.

[22] Bianchi, Daniele, Francesco Nasuti, and Emanuele Martelli. Navier-Stokes simulations of hypersonic flows with coupled graphite ablation, Journal of Spacecraft and Rockets 47.4 (2010): 554-562.

[23] Estorf, M., Radespiel, R., Schneider, S., Johnson, H., and Hein, S. Surface-pressure measurements of second-mode instability in quiet hypersonic flow, AIAA Paper 2008-1153, 46th AIAA Aerospace Sciences Meeting and Exhibit, Reno, Nevada, 2008.

[24] McKenzie, J. F., and K. O. Westphal. "Interaction of linear waves with oblique shock waves." Physics of Fluids 11.11 (1968): 2350-2362.

[25] De Tullio, Nicola, and Neil D. Sandham. Influence of boundary-layer disturbances on the instability of a roughness wake in a high-speed boundary layer, Journal of Fluid Mechanics 763 (2015): 136-165. 